\title{
Retronasal aroma allows feature extraction from taste of a traditional Japanese confection
}

\author{
Naomi Gotow ${ }^{1}$, Takefumi Kobayashi ${ }^{2}$ and Tatsu Kobayakawa ${ }^{1 *}$
}

\begin{abstract}
Background: Common foods consist of several taste qualities. Consumers perceive intensity of a particular taste quality after noticing it among other taste qualities when they eat common foods. We supposed that while one is eating the facility for noticing a taste quality present in a common food will differ among taste qualities which compose the common food. We, therefore, proposed a new measurement scale for food perception named 'noticeability'. Furthermore, we found that consumers' food perceptions to common foods were modified by retronasal aroma. In this study, in order to examine whether retronasal aroma affects the relationship between noticeability and perceived intensity for taste, we evaluated participants for noticeability and perceived intensity of five fundamental taste qualities (sweetness, saltiness, sourness, bitterness, and umami) under open and closed nostril conditions using one of the most popular traditional Japanese confections called 'yokan'.

Results: The taste quality showed that the highest noticeability and perceived intensity among five fundamental taste qualities for yokan was sweetness, independent of the nostril condition. For sweetness, a significant decrease of correlation between noticeability and perceived intensity was observed in response to retronasal aroma. On the other hand, for umami, correlation between noticeability and perceived intensity significantly increased with retronasal aroma.
\end{abstract}

Conclusions: As the retronasal aroma of yokan allowed feature extraction from taste by Japanese consumers, we reconfirmed that consumers' food perceptions were modified by the retronasal aroma of a common food.

Keywords: Common food, Consumer, Feature extraction, Five fundamental taste qualities, Japanese confection, Noticeability, Perceived intensity, Retronasal aroma

\section{Background}

Cognitive processes that govern consumers' preferences for various foods are complicated. Many studies have examined consumers' perceptions of common foods, including evaluations of hedonics [1-4], palatability [5,6], and satiation [7].

Clearly, gustation is an important sensory modality for food perception. Taste buds distributed throughout the tongue detect chemical substances inside the oral cavity, converting the stimuli into electric signals in taste cells. The signals are then transmitted via the gustatory nerve to the cortical gustatory areas $[8,9]$, and are processed to produce psychophysical evaluations of taste, such as

\footnotetext{
* Correspondence: kobayakawa-tatsu@aist.go.jp

'Human Technology Research Institute, National Institute of Advanced Industrial Science and Technology (AIST), Tsukuba Central 6, 1-1-1 Higashi, Tsukuba, Ibaraki 305-8566, Japan

Full list of author information is available at the end of the article
}

perceived intensity $[10,11]$. Such psychophysical evaluation of taste is affected not only by the physiological aspect, such as amplitude of the signals in gustatory nerve from taste bud [12], but also by the psychological aspect, such as attention [13-15].

Chemical substances detected by taste cells have been physiologically and psychologically classified into five fundaments taste qualities: sweetness, saltiness, sourness, bitterness, and umami [16]. In sensory evaluation using common foods, perceived intensity of five fundamental taste qualities is frequently measured. For example, Ali and colleagues [17] asked recreational exercisers to evaluate perceived intensity of sweetness and saltiness using three sport drinks with different amounts of carbohydrate, electrolyte and water, in order to examine how perceived intensity changed before, during and after running on a treadmill. Their results indicated that perceived intensity of sweetness increased during exercise as compared to 
non-exercise, but that perceived intensity of saltiness decreased more during exercise than non-exercise. Bossola and colleagues [18] examined the effects of gastrointestinal cancer on taste of common foods by asking patients and healthy volunteers to evaluate perceived intensity of the sweetness of a black currant drink with additional sucrose, the sourness of lemonade with additional citric acid, the saltiness of unsalted tomato juice with additional $\mathrm{NaCl}$, and the bitterness of tonic water with additional urea. Their results indicated that values of perceived intensity were similar between patients and healthy volunteers, so that decrease of perceived intensity caused by disease was not observed. Accordingly, measurement of perceived intensity of taste was effective in clarifying the sensory properties of consumers during food intake.

The most frequent phenomenon of multisensory integration would be consumers' food perception in daily life [19]. Mr. Mitsutomo Kurokawa - the 16th head of family and a former president of the Toraya Confectionery Company Limited, which is a traditional Japanese confectionary established about five hundred years ago in Kyoto - once stated 'Wagashi is the art of the five senses' $[20,21]$. The syllable ' $w a$ ' means things Japanese and 'gashi', sequential voicing 'kashi', means confection. As symbolized by Mr. Kurokawa's words, consumers perceive a common food by integrating signals from various sensory systems when they eat the food, such as wagashi. Thus, multiple sensations affect food perception. In particular, among these sensations, taste and retronasal aroma play significant roles in the occurrence of unified oral sensation $[22,23]$. Although various data suggest that cognitive processes rather than peripheral sensory processing underlie the perceptual whole between taste and retronasal aroma [24], the precise neural mechanisms have not been elucidated $[22,25,26]$.

Taste interacts with retronasal aroma deeply. Many studies have shown that retronasal aroma enhances perceived intensity of taste [27-30]. Stevenson and colleagues [30] examined perceived intensity of sweetness in sucrose solutions containing different odorants. The authors found that some odorants, such as caramel, strawberry, and maracuja, significantly enhanced the sweetness of the sucrose solution. Schifferstein and Verlegh [29] had participants with open or closed nostrils evaluate perceived intensity of sweetness using sucrose solution containing a strawberry odorant. The results indicated the strawberry odorant in the sucrose solution significantly increased perceived intensity of sweetness when nostrils were open, whereas the effect was not observed when nostrils were closed. Additionally, an evaluation of the sweetness of whipped cream with additional sucrose and a strawberry odorant by Frank and Byram [27] produced similar results to those shown by Schifferstein and Verlegh [29]. These results suggested that association learning of taste and odor inhibited to perceive olfaction and gestation independently, so that the confusion occurred between both modalities [31].

Many studies on enhancement of taste by retronasal aroma were conducted in terms of attention [24,32-36]. These studies found that the manipulation of directing attention by instruction or cognitive task affects occurrence of enhancement of taste by retronasal aroma. Frank and colleagues [33] conducted the evaluation of perceived intensity for sucrose solution with strawberry odor using three different instructions. One instruction was to evaluate perceived intensity of sweetness for its solution. Another instruction was to evaluate perceived intensities of sweetness, sourness, and fruitiness for its solution. The other was to evaluate all over perceived intensity for its solution, and then to break down all over intensity into perceived intensities of sweetness, saltiness, sourness, fruitiness, and other taste. The results indicated that perceived intensity of sweetness for its solution decreased with more evaluation items. In other words, whereas the evaluation of perceived intensity of only sweetness caused taste enhancement by retronasal aroma, such an enhancement was not observed in cases where all over intensity was broken down into several attributes. Furthermore, a similar result was observed in a solution of quinine hydrochloride with lemon or almond odor. Thus, these results showed that effect of instruction on occurrence of enhancement of taste by retronasal aroma was not observed only in sweetness. Prescott and colleagues [24] conducted evaluations of perceived intensity of sweetness for a sucrose solution with prune or water chestnut odors, before or after a discrimination task using two different instructions. Each participant was presented either prune odor or water chestnut odor in the discrimination task. In the discrimination task participants who asked to take a synthetic strategy selected the solution with the strongest intensity of all over flavor among three solutions. On the other hand, participants who asked to take an analytic strategy performed the discrimination task, which consisted of two sessions. One session was to select the solution with the strongest sweetness among three solutions, and the other session was to select the solution with strongest flavor among three solutions. Participants of the analytic strategy group were given additional information that the solutions contained sucrose and odor. As a result, whereas enhancement of taste by retronasal aroma occurred in the synthetic strategy group, this was not observed in the analytic strategy group. Furthermore, Bigham and colleagues [37], who investigated the effect of maltol to sweetness in sucrose solution using trained panels and untrained panels, reported that untrained panels indicated significant enhancement of sweetness in the sucrosemaltol solution whereas trained panels did not perceive the sucrose-maltol solution to be significantly sweeter than an 
equivalent concentration of sucrose solution. One explanation they offer for this result is that trained panels might attend to sweetness in the solution after ignoring the effect of odor. In other words, the strategy that panelists adopt during sensory evaluation might change spontaneously from an analytic strategy to a synthetic strategy by training, and trained panels found no enhancement of sweetness in the solution by maltol [24]. Based on these studies, we presumed that attention might play an important role in food recognition, which taste and odor affected greatly.

As described above, common foods consist of several taste qualities. Gustatory processing of common foods will differ from that observed in experimental evaluations of taste solutions containing a single chemical substance, such as sucrose or citric acid [38-40]. For example, consumers perceive the value of sensory evaluation for a particular taste quality after they notice it among other taste qualities. In other words, there seems to be a temporal order on psychological processing between noticing a taste quality included in common food (the advance processing) and evaluating its perceived intensity (the subsequent processing).

We supposed that the facility for noticing a taste quality present in a common food while one is eating will differ among taste qualities that compose the common food. Based on this speculation and, in order to examine the consumers' perception of common foods from the point of view of attention, we consider that it should be important to assess not only conventional measurement scales, such as perceived intensity, but also a new scale, which reflected the facility for noticing a certain taste quality during food intake. In this study, therefore, we propose a new measurement scale named 'noticeability' for each taste quality of common foods.

In this study, participants evaluated noticeability and perceived intensity of sweetness, saltiness, sourness, bitterness, and umami using one of the most popular traditional Japanese confections 'yokan.' As described above, retronasal aroma as well as taste, influences consumers' perceptions of common foods [21,27]. Therefore, participants in this study evaluated yokan with open or closed nostrils. This allowed us to clarify the effects of the retronasal aroma of yokan on the feature extraction of taste of it.

\section{Methods}

\section{Participants}

This study was conducted in accordance with the revised Declaration of Helsinki. All procedures in this study were approved by the ethics committee for ergonomic experiments from the National Institute of Advanced Industrial Science and Technology (AIST) in Japan. We explained the experimental approach and ingredients of yokan to the participants before the evaluations, obtained written consent, and informed the participants that they could stop participating at any time. Potential participants who were allergic to any of the yokan ingredients were advised not to perform the evaluation.

Ninety students from Bunkyo Gakuin University (53 women and 37 men) between the ages of 19 and 26 years old (mean age \pm SD, $20.3 \pm 1.0$ years) who applied for a special lecture at AIST participated in this study.

\section{Materials}

We used yokan ('Yoru no ume', Toraya Confectionary Co., Ltd, Tokyo, Japan), one of the most popular traditional Japanese confections. Yokan is neither a staple food nor a regularly stocked food in Japan, and Japanese consumers often eat yokan at tea time or after a meal with green tea. Yokan is also served as a confection at tea ceremonies. To the best of our knowledge, this is the first psychophysical study of the taste of yokan.

The ingredients of yokan are simply red azuki beans, agar and sugar. To make yokan, azuki bean paste was made by straining red azuki beans that had been boiled until they became soft. Next, a pot containing agar and water was boiled until the agar melted completely. Sugar and azuki bean paste was added to the pot, and the mixture was reduced via boiling. The resulting hot reduction was poured into a loaf pan, and cooled in a refrigerator. After the material solidified, it was removed from the pan and cut to a thickness of 1 to $2 \mathrm{~cm}$ with a cooking knife. Japanese consumers generally eat yokan at room temperature while cutting it into bite-size pieces with a large traditional Japanese toothpick called a 'kuromoji.'

During the pilot study we asked another group of students who did not participate in the evaluation of noticeability and perceived intensity to fill out a questionnaire about their impression of and preference for yokan, as well as how often and under what circumstances they eat yokan. The 72 students made up a psychology class at Bunkyo Gakuin University, and the 71 students of them (44 women and 27 men; one woman who had not eaten yokan was excluded) participated this pilot study between 19 and 30 years old (mean \pm SD, $20.1 \pm 1.6$ years). After we instructed participants to provide their impressions of yokan while focusing on the taste, we asked them for free descriptions. Participants also used a scale to note their preference for yokan from -10 (dislike a lot) to 10 (like a lot), and the scale had 19 vertical lines at regular interval between both ends; participants were asked 'How much do you like or dislike yokan?' and were instructed to mark the appropriate point on the scale, potentially including the spaces between the vertical label lines on the scale. For the frequency of yokan consumption, participants used a five-step ordinal scale: 'almost never,' 'rarely,' 'occasionally', 'sometimes', and 'often.' Participants were asked 'How often do you eat yokan?' and participants who had eaten yokan 
more than 'almost never' were asked to describe under what circumstances they eat yokan.

In the participants' impressions of yokan, the word 'sweet' appeared in the descriptions of 57 of the 71 students (80.3\%). The preference value for yokan was $2.9 \pm$ 5.3 (mean value $\pm \mathrm{SD}$ ), which indicted that this young Japanese population generally liked yokan. Forty-three students $(60.6 \%)$ ate yokan 'almost never' or 'rarely', whereas 28 students (39.4\%) consumed yokan 'occasionally', 'sometimes', or 'often.' Thus, the young Japanese population did not eat yokan frequently. Many participants stated that they ate yokan when they obtained it as a gift or visited their grandparents.

\section{Procedure}

Approximately 1 hour prior to the start of the evaluation, yokan was cut into bite-sized pieces $(3 \mathrm{~cm} \times 2 \mathrm{~cm} \times$ $1 \mathrm{~cm}$ cubes), and two pieces were placed on each small disposable polystyrene plate. To prevent the surface of the yokan from drying, we covered the plates with polyvinylidene chloride wrap ('Saran Wrap", Asahi Kasei Home Products Corporation, Tokyo, Japan). We arranged plates, bottled mineral water ('Evian', Danon Waters of Japan Co., Tokyo, Japan), toothpicks made of birch, and questionnaires on the meeting tables before the participants entered the room. Yokan was presented at room temperature (26 to $27^{\circ} \mathrm{C}$ ).

We divided the participants into two groups of 40 and 50 participants, and gathered each group in a meeting room. The meeting tables were $180-\mathrm{cm}$ wide, and two participants sat at each table with an empty seat between them. The width of the aisles between the rows of tables was approximately $1 \mathrm{~m}$. Before the evaluation began, we closed the door of room, closed blinds on the windows, and turned fluorescent lights on.

Before the evaluation began, we provided participants with instructions about the experiment using a slide presentation, and described the same instructions provided at the top of each questionnaire. We instructed participants not to consult with other participants during the experiment, and the evaluation was performed simultaneously. To help participants who did not understand the instructions, two experimenters and six assistants stationed in each room walked around the room to monitor the participants.

Each participant tasted yokan under open and closed nostril conditions. In examinations of single tastants, such as sucrose or citric acid, it may be possible to alter perceived intensity of retronasal aroma based on the amount of odorant added. On the other hand, it may be difficult to completely remove retronasal aroma from many common foods. Murphy and Cain [41] reported that retronasal aroma was not perceived when the nostrils are closed even at higher levels of added odorant.
To eliminate retronasal aroma of yokan, we had participants pinch their nostrils with their fingers during the evaluation under the closed nostril condition (see Experiment 4 in Frank and Byram, [27]). The order of the open and closed nostril conditions was set randomly among the participants.

\section{Open nostril condition}

In this condition, participants performed the evaluation with open nostrils. Participants were instructed to rinse the insides of their mouths with mineral water and swallow. Each participant then used a toothpick to put one piece of yokan into her or his mouth. After sufficient mastication and tasting, the yokan was swallowed. Immediately after swallowing, participants used a scale described below to evaluate noticeability and perceived intensity of sweetness, saltiness, sourness, bitterness, and umami.

The scale for noticeability was labeled from 0 (very easy) to 6 (very difficult), and was drawn using five vertical lines at regular intervals between both ends; participants were asked 'How easy or difficult was it to notice each taste quality?' The scale for perceived intensity was labeled 0 (tasteless), 1 (barely detectable), 2 (weak), 3 (easily detectable), 4 (strong), and 5 (very strong) [42]; participants were asked 'What was the perceived intensity of each taste quality?' Participants were instructed to mark the appropriate point on the scale, potentially including the spaces between the vertical label lines.

\section{Closed nostril condition}

In this condition, participants performed the evaluation while they had their nostrils closed. After rinsing out their mouths with mineral water, the participants sufficiently masticated and tasted one piece of yokan while pinching both sides of their noses with their thumbs and index fingers to close their nostrils. Immediately after swallowing the yokan, the participants rated noticeability and perceived intensity of sweetness, saltiness, sourness, bitterness, and umami using the same scales described for the evaluation under open nostril condition.

\section{Statistical analysis}

Three participants who did not eat yokan, and one participant who had missing evaluation values were not included in the following analyses. Therefore, evaluation values were obtained from 86 students (50 women and 36 men) between 19 and 26 years old (mean age \pm SD, $20.3 \pm 1.1$ years), and were analyzed using statistical analysis software ('SPSS 10.0), SPSS Japan Inc., Tokyo, Japan).

To examine the effect of retronasal aroma on feature extraction from yokan based on the evaluation of five fundamental taste qualities, a two-way repeatedmeasures analysis of variance (ANOVA) was performed 
for noticeability or perceived intensity with the nostril condition and taste quality as factors. Simple effect tests and multiple comparisons among taste qualities using Ryan's method were performed based on the significance of the results obtained with the ANOVA.

To examine the effect of retronasal aroma on feature extraction from yokan based on the relationship between noticeability and perceived intensity for five fundamental taste qualities, we calculated Spearman's rank correlation coefficients for each taste quality when the participants' nostrils were open or closed. Correlation coefficients for each open and closed nostril condition were then compared based on Fisher's z-transformation, for each taste quality, respectively.

\section{Results}

\section{Noticeability}

Table 1 shows noticeability of each taste quality of yokan under open and closed nostril conditions. Two-way repeated-measures ANOVA demonstrated significance in the main effects of the nostril condition $(F(1,85)=32.11$, $P<0.001)$ and taste quality $(F(4,340)=124.29, P<0.001)$, and interaction between the nostril condition and taste quality $(F(4,340)=9.54, P<0.001)$. We conducted simple effect tests for interaction between nostril condition and taste quality, and found significance in the simple main effects of the nostril condition for sweetness $(F(1,425)=$ 64.92, $P<0.001)$, umami $(F(1,425)=16.28, P<0.001)$, saltiness $(F(1,425)=10.35, P<0.01)$, and those of the taste quality under open $(F(4,680)=126.10, P<0.001)$ and closed nostril conditions $(F(4,680)=67.49, P<0.001)$. Multiple comparisons between paired taste qualities under open nostril conditions revealed significant differences for all pairs except the combination of sourness and bitterness $(P<0.05)$. On the other hand, multiple comparisons between paired taste qualities under closed nostril condition revealed significant difference for six pairs except the combination of saltiness and umami, saltiness and sourness, saltiness and bitterness, and sourness and bitterness $(P<0.05)$. We confirmed that the highest noticeability values for yokan were for sweetness, independent of the nostril condition. The taste quality associated with the second highest noticeability value was umami, followed by saltiness, whereas it was difficult to attend to sourness and bitterness. Furthermore, we observed significant enhancements of noticeability of sweetness, umami, and saltiness in response to retronasal aroma.

\section{Perceived intensity}

Table 1 shows perceived intensity of each taste quality of yokan under open and closed nostril conditions. Twoway repeated-measures ANOVA demonstrated significance in the main effects of the nostril condition $(F(1,85)=$ 47.58, $P<0.001)$ and taste quality $(F(4,340)=271.75$, $P<0.001)$, and interaction between the nostril condition and taste quality $(F(4,340)=19.50, P<0.001)$. We conducted simple effect tests for interaction between nostril condition and taste quality, and found significance in the simple main effects of the nostril condition for sweetness $(F(1,425)=105.88, P<0.001)$ and umami $(F(1,425)=31.62, P<0.001)$, and those of the taste quality under open $(F(4,680)=270.28, P<0.001)$ and closed nostril conditions $(F(4,680)=144.62, P<0.001)$. Multiple comparisons between paired taste qualities under open and closed nostril conditions revealed significant differences for all pairs except the combination of sourness and bitterness under both nostril conditions $(P<0.05)$. We confirmed that the highest perceived intensity values for yokan were for sweetness, independent of the nostril condition. The taste quality associated with the second highest perceived intensity value was umami, followed by saltiness, whereas sourness and bitterness were not perceived as marked components of the yokan. Furthermore, we observed significant enhancements of perceived intensity of sweetness and umami in response to retronasal aroma.

\section{Correlation between noticeability and perceived intensity}

We created scatter diagrams for five fundamental taste qualities for the open and closed nostril conditions by plotting noticeability and perceived intensity along horizontal and vertical axes, and appending Spearman's rank correlation coefficients, respectively (Figure 1). Comparisons

Table 1 Noticeability and perceived intensity of each taste quality under open and closed nostril conditions

\begin{tabular}{|c|c|c|c|c|}
\hline \multirow[t]{3}{*}{ Taste quality } & \multicolumn{2}{|c|}{ Noticeability } & \multicolumn{2}{|c|}{ Perceived intensity } \\
\hline & Open nostril & Closed nostril & Open nostril & Closed nostril \\
\hline & Mean \pm SD & Mean \pm SD & Mean \pm SD & Mean \pm SD \\
\hline Sweetness & $5.61 \pm 0.91$ & $4.07 \pm 2.05$ & $3.98 \pm 0.55$ & $2.92 \pm 1.26$ \\
\hline Umami & $2.52 \pm 2.27$ & $1.75 \pm 1.96$ & $1.86 \pm 1.61$ & $1.29 \pm 1.29$ \\
\hline Saltiness & $1.97 \pm 1.87$ & $1.35 \pm 1.77$ & $0.86 \pm 0.88$ & $0.67 \pm 0.84$ \\
\hline Sourness & $1.17 \pm 1.91$ & $0.83 \pm 1.74$ & $0.36 \pm 0.60$ & $0.30 \pm 0.59$ \\
\hline Bitterness & $1.22 \pm 1.97$ & $0.86 \pm 1.75$ & $0.31 \pm 0.59$ & $0.19 \pm 0.39$ \\
\hline
\end{tabular}

SD, standard deviation. 


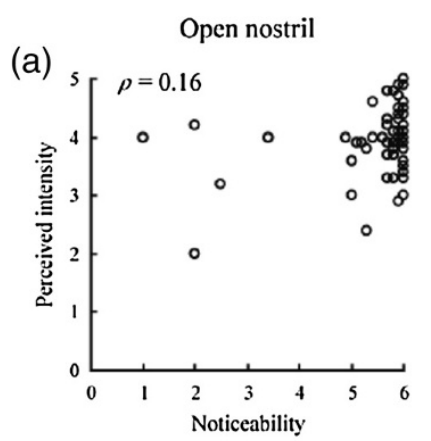

Closed nostril
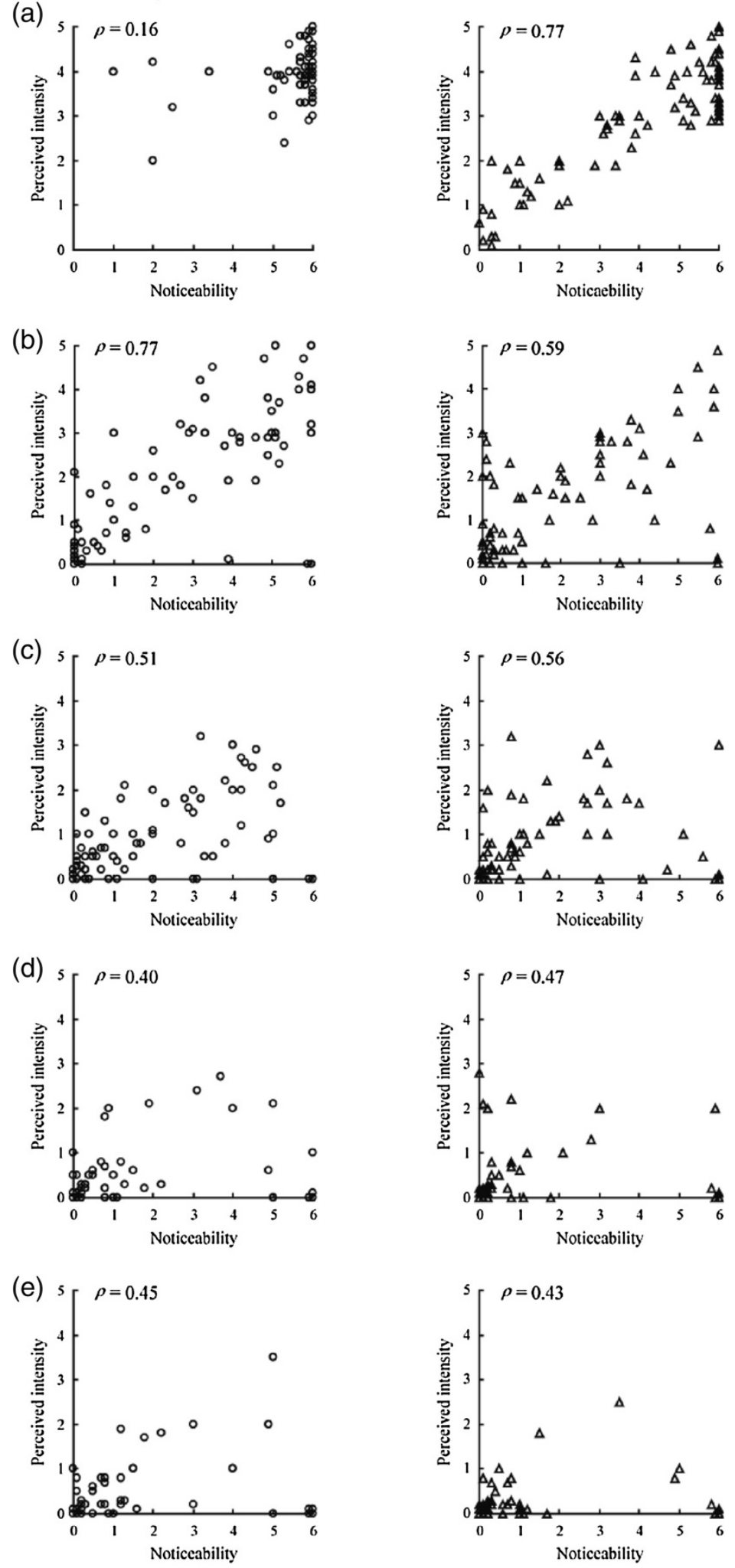

Figure 1 (See legend on next page.) 
(See figure on previous page.)

Figure 1 Relationship between noticeability and perceived intensity for each taste quality under open and closed nostril conditions. Scatter diagrams for each taste quality show plots of noticeability and perceived intensity along the horizontal and vertical axes, respectively: (a) sweetness, (b) umami, (c) saltiness, (d) sourness, and (e) bitterness. The diagrams representing open and closed nostril conditions are shown in the left and right columns, respectively. The $\rho$ values shown in each diagram are Spearman's rank correlation coefficients between noticeability and perceived intensity for taste qualities obtained when the participants' nostrils were open or closed.

between correlation coefficients based on Fischer's $z$ transformation revealed significant differences for sweetness $(z=-5.53, P<0.001)$ and umami $(z=2.20, P<0.05)$. The correlation coefficient for sweetness when the nostrils were closed was significantly higher than when the nostrils were open. On the other hand, the correlation coefficient for umami when the nostrils were open was significantly higher than when the nostrils were closed. Furthermore, non-significant differences were revealed in the comparisons between the correlation coefficients for saltiness $(z=-0.48, n$. s. $)$, sourness $(z=-0.61, n$. s. $)$, or bitterness $(z=0.13, n$. s. $)$ when the nostrils were open or closed.

\section{Discussion}

We used one of the most traditional Japanese confections called yokan to evaluate noticeability and perceived intensity of five fundamental taste qualities (sweetness, saltiness, sourness, bitterness, and umami) while participants had their nostrils open or closed.

\section{Enhancement of taste by retronasal aroma}

In order to examine the effect of retronasal aroma on feature extraction from yokan based on the evaluation of five fundamental taste qualities, we compared noticeability and perceived intensity of the five fundamental taste qualities while the participants had their nostrils open and closed. The taste quality with the highest noticeability and perceived intensity value was sweetness, followed by umami and saltiness, whereas sourness and bitterness were hardly noticed and perceived. Of note, Japanese consumers often state that yokan is 'sweet' [43]. Furthermore, more than $80 \%$ of participants in our pilot study described yokan as 'sweet' on the questionnaire about their impression of yokan. Thus, these Japanese consumers' impressions of yokan were substantiated by our evaluation of noticeability and perceived intensity.

Significant effects of retronasal aroma on taste in the noticeability evaluation were observed for three taste qualities, such as sweetness, umami, and saltiness. Meanwhile, significant effects of retronasal aroma on taste in the perceived intensity evaluation were observed for two taste qualities, such as sweetness and umami. Although this implies that the measurement scale that reflects an enhancement of taste by retronasal aroma with greater sensitivity might be noticeability rather than perceived intensity, this speculation would need examining carefully in future. In contrast to our results, Green and colleagues [44] did not observe a retronasal aroma-induced enhancement of taste during evaluations of perceived sweetness intensity of custard containing a vanilla odorant. In that study, participants performed the evaluation after they spat the custard from their mouths, while our participants evaluated after swallowing the yokan. Frank and colleagues [28] examined how differences in evaluation procedures affected enhancement of taste by retronasal aroma using sucrose solutions with additional strawberry odorant. Evaluations in which participants swallowed the sucrose solutions were more consistent and stable than those in which participants held the sucrose solutions in their mouths and then spat them out, although significant retronasal aromainduced enhancements of taste were observed with both evaluation procedures. Therefore, the relationships between taste qualities and retronasal aroma may change depending on the details of the experimental procedures, such as spitting out or swallowing.

Azuki bean paste, which contains sugar and boiled red azuki beans, is frequently used in traditional Japanese confections. Consumers familiar with traditional Japanese confections likely think that taste and retronasal aroma of the azuki bean paste are congruous. Many studies have shown that this congruency in the consumers' experience is necessary for the enhancement of taste by retronasal aroma [27-29,45]. Furthermore, because pair-presentation of taste and odor occurs in consumers' everyday lives, association between taste and odor might succeed by implicit learning. Stevenson and colleagues $[46,47]$, who repeated presentation of sucrose solution with lychee or water chestnut odors, reported that evaluation values of sweetness for odor increased significantly irrespective of whether or not participants could be aware of pair-presentation of sucrose and odor. Based on such enhancement of taste attribution on odor by implicit learning, enhancement of taste by retronasal aroma might also succeed implicitly. In other words, we speculate that the significant enhancement of taste by the retronasal aroma of yokan may not be observed in consumers who are unfamiliar with azuki bean paste.

\section{Modification of relationship between noticeability and perceived intensity by retoronasal aroma}

We examined the effect of retronasal aroma on feature extraction from yokan based on the relationship between noticeability and perceived intensity for five fundamental taste qualities. Correlation between noticeability 
and perceived intensity for sweetness under closed nostril condition was significantly higher than under open nostril condition. On the other hand, correlation between noticeability and perceived intensity for umami under open nostril condition was significantly higher than under closed nostril condition.

A strong correlation between noticeability and perceived intensity for sweetness was observed under closed nostril conditions where participants were unable to obtain olfactory information of yokan, whereas a weak correlation between both scales for sweetness was shown under open nostril conditions where participants were able to obtain olfactory information of it. We considered that the low correlation between noticeability and perceived intensity for sweetness under the open nostril condition was because the majority of participants who evaluated noticeability as 'very easy' indicated perceived intensity that ranged from middle to high values on the scale. Based on these consequences, availability of olfactory information would be necessary for a correlating decrease between noticeability and perceived intensity in common foods. Furthermore, we currently have four hypotheses concerning necessary conditions other than olfactory information. The first hypothesis is that sweetness may tend to be a necessary condition regardless of perceived intensity value. For example, the organism may be inherently more sensitive to sugar, because sugar is an important energy resource for human [48-50]. The second hypothesis is that taste quality with the highest perceived intensity may be a necessary condition. For example, consumers would be able to identify easily the taste of salt in broth or the taste of sweetener in a beverage when they eat very salty soup or drink very sweet tonic water [13]. The third hypothesis is that the taste quality that attracts the attention most spontaneously will become a necessary condition. For instance, if consumers perceive a very unpleasant taste when eating a food, this taste would probably be easily noticed regardless of its perceived intensity value. The fourth hypothesis is that high familiarity with a common food may be a necessary condition. A common food which consumers eat high frequently in their everyday lives leads implicitly to association learning between taste and odor, and such learning is regarded as a precise example of learned synesthesia [47]. We considered that familiarity with a common food might relate deeply to establishment of implicit association learning between taste and odor. Accordingly, if consumers with a food culture that differs from the food culture of the Japanese eat yokan under open nostril condition, a decrease in correlation between noticeability and perceived intensity for sweetness might not be observed. The validities of theses hypotheses should be examined by evaluating noticeability and perceived intensity of taste qualities using various consumers with different food cultures and various common foods other than yokan, and identifying conditions which are observed that decrease the correlation between these measurement scales.

\section{Conclusions}

In gustatory information processing on a common food, consumers notice a taste quality among other taste qualities, and then they evaluate its perceived intensity. Furthermore, if consumers eat a common food, the facility for noticing that a taste quality exists in the food will differ among the taste qualities that compose the food. Based on this speculation, we proposed a new measurement scale named 'noticeability' of each taste quality.

Participants in this study evaluated noticeability and perceived intensity of five fundamental taste qualities of yokan (sweetness, saltiness, sourness, bitterness, and umami) under open or closed nostril conditions using one of the most popular traditional Japanese confections 'yokan'. Most noticeability values for sweetness increased to nearly maximum in response to the retronasal aroma of yokan, independent of the perceived intensity value for sweetness, such that the correlation between noticeability and perceived intensity significantly decreased. On the other hand, for umami, the correlation between noticeability and perceived intensity significantly increased in response to the retronasal aroma of yokan. On the basis that retronasal aroma of yokan allows feature extraction from taste of it in Japanese consumers, we have reconfirmed that consumers' food perception is modified by the retronasal aroma of a common food.

\section{Competing interests}

The authors declare that they have no competing interests.

\section{Authors' contributions}

NG conceived of the study, participated in its design, performed the statistical analysis, and drafted the manuscript. TK, who was second author, participated in the design and coordination of the study. TH, who was last and corresponding author, conceived of the study, participated in its design and coordination, and supervised the drafting of the manuscript. All authors read and approved the final manuscript.

\section{Acknowledgements}

The authors are deeply grateful to Mr. Takuya Yokoi and Ms. Hiroko Hara (Toraya Research Institute, Toraya Confectionary Co., Tokyo, Japan) who gave us the idea for a psychophysics study using wagashi. This study was partially supported by the Sapporo Bioscience Foundation.

\section{Author details}

${ }^{1}$ Human Technology Research Institute, National Institute of Advanced Industrial Science and Technology (AIST), Tsukuba Central 6, 1-1-1 Higashi, Tsukuba, Ibaraki 305-8566, Japan. ${ }^{2}$ The Faculty of Human Studies, Bunkyo Gakuin University, 1196 Kamekubo, Fujimino, Saitama 356-8533, Japan.

Received: 23 May 2013 Accepted: 19 September 2013

Published: 30 September 2013 


\section{References}

1. Koskinen S, Kälviäinen N, Tuorila H: Flavor enhancement as a tool for increasing pleasantness and intake of a snack product among the elderly. Appetite 2003, 41:87-96.

2. Kremer S, Bult JH, Mojet J, Kroeze JH: Compensation for age-associated chemosensory losses and its effect on the pleasantness of a custard dessert and a tomato drink. Appetite 2007, 48:96-103.

3. Kremer S, Bult JH, Mojet J, Kroeze JH: Food perception with age and its relationship to pleasantness. Chem Senses 2007, 32:591-602.

4. Tuorila H, Niskanen N, Maunuksela E: Perception and pleasantness of a food with varying odor and flavor among the elderly and young. J Nutr Health Aging 2001, 5:266-268.

5. Bolhuis DP, Lakemond CM, de Wijk RA, Luning PA, de Graaf C: Effect of salt intensity on ad libitum intake of tomato soup similar in palatability and on salt preference after consumption. Chem Senses 2010, 35:789-799.

6. Forde $C G$, Cantau B, Delahunty CM, Elsner RJ: Interactions between texture and trigeminal stimulus in a liquid food system: effects on elderly consumers preferences. J Nutr Health Aging 2002, 6:130-133.

7. Ruijschop RM, Boelrijk AE, Burgering MJ, de Graaf C, Westerterp-Plantenga MS: Acute effects of complexity in aroma composition on satiation and food intake. Chem Senses 2010, 35:91-100.

8. Kobayakawa T, Endo H, Ayabe-Kanamura S, Kumagai T, Yamaguchi Y, Kikuchi Y, Takeda T, Saito S, Ogawa H: The primary gustatory area in human cerebral cortex studied by magnetoencephalography. Neurosci Lett 1996, 212:155-158.

9. Kobayakawa T, Ogawa H, Kaneda H, Ayabe-Kanamura S, Endo H, Saito S: Spatio-temporal analysis of cortical activity evoked by gustatory stimulation in humans. Chem Senses 1999, 24:201-209.

10. Small DM, Gregory MD, Mak YE, Gitelman D, Mesulam MM, Parrish T: Dissociation of neural representation of intensity and affective valuation in human gustation. Neuron 2003, 39:701-711.

11. Kobayakawa T, Saito S, Gotow N: Temporal characteristics of neural activity associated with perception of gustatory stimulus intensity in humans. Chemosensory Perception 2012, 5:80-86.

12. Spetter MS, Smeets PA, de Graaf C, Viergever MA: Representation of sweet and salty taste intensity in the brain. Chem Senses 2010, 35:831-840.

13. Marks LE: The role of attention in chemosensation. Food Quality and Preference 2002, 14:147-155.

14. Marks LE, Wheeler ME: Attention and the detectability of weak taste stimuli. Chem Senses 1998, 23:19-29.

15. Marks LE, Wheeler ME: Focused attention and the detectability of weak gustatory stimuli. Empirical measurement and computer simulations. Ann N Y Acad Sci 1998, 855:645-647.

16. Brand JG: Biophysics of taste. In Tasting and smelling. Edited by Beauchamp GK, Bartoshuk L. San Diego: Academic; 1997:1-24.

17. Ali A, Duizer L, Foster K, Grigor J, Wei W: Changes in sensory perception of sports drinks when consumed pre, during and post exercise. Physiol Behav 2011, 102:437-443.

18. Bossola M, Cadoni G, Bellantone R, Carriero C, Carriero E, Ottaviani F, Borzomati D, Tortorelli A, Doglietto GB: Taste intensity and hedonic responses to simple beverages in gastrointestinal cancer patients. J Pain Symptom Manage 2007, 34:505-512.

19. Zampini M, Spence C: Assessing the role of visual and auditory cues in multisensory perception of flavor. In The Neural Bases of Multisensory Processes. Edited by Murray MM, Wallace MT. Boca Raton: CRC Press; 2012.

20. Toraya confectionery: The art of the five senses. http://www.toraya-group.co. jp/english/wagashi/art.html.

21. Kurokawa M: Toraya Confectionay Co., Ltd.: Five Hundred Years Walked with Japanese Confections [in Japanese, Toraya: Wagashi to ayunda gohyakunen]. Tokyo: Shinchosya Publishing; 2005 .

22. Dalton P, Doolittle N, Nagata H, Breslin PS: The merging of the senses: integration of subthreshold taste and smell. Nat Neurosci 2000, 3:431-432.

23. Lim J, Johnson MB: The role of congruency in retronasal odor referral to the mouth. Chem Senses 2012, 37:515-522.

24. Prescott J, Johnstone V, Francis J: Odor-taste interactions: effects of attentional strategies during exposure. Chem Senses 2004, 29:331-340.

25. Djordjevic J, Zatorre RJ, Jones-Gotman M: Odor-induced changes in taste perception. Exp Brain Res 2004, 159:405-408.

26. Sakai N, Kobayakawa T, Gotow N, Saito S, Imada S: Enhancement of sweetness ratings of aspartame by a vanilla odor presented either by orthonasal or retronasal routes. Percept Mot Skills 2001, 92:1002-1008.
27. Frank RA, Byram J: Taste-smell interactions are tastent and odorant depent. Chem Senses 1988, 13:445-455.

28. Frank RA, Ducheny K, Mize SJS: Strawberry odor, but not red color, enhances the sweetness of sucrose solutions. Chem Senses 1989, 14:371-377.

29. Schifferstein HNJ, Verlegh PWJ: The role of congruency and pleasantness in odor-induced taste enhancement. Acta Psychol (Amst) 1996, 94:87-105.

30. Stevenson RJ, Prescott J, Boakes RA: Confusing tastes and smells: how odours can influence the perception of sweet and sour tastes. Chem Senses 1999, 24:627-635.

31. Rozin P: "Taste-smell confusions" and the duality of the olfactory sense. Perception and Psychophysics 1982, 31:397-401.

32. Clark CC, Lawless HT: Limiting response alternatives in time-intensity scaling: an examination of the halo-dumping effect. Chem Senses 1994, 19:583-594.

33. Frank RA, van der Klaauw NJ, Schifferstein HN: Both perceptual and conceptual factors influence taste-odor and taste-taste interactions. Percept Psychophysics 1993, 54:343-354.

34. Labbe D, Martin N: Impact of novel olfactory stimuli at supra and subthreshold concentrations on the perceived sweetness of sucrose after associative learning. Chem Senses 2009, 34:645-651.

35. Prescott J: Flavour as a psychological construct: implications for perceiving and measuring the sensory qualities of foods. Food Quality and Preference 1999, 10:349-356.

36. Prescott J, Murphy S: Inhibition of evaluative and perceptual odour-taste learning by attention to the stimulus elements. Q J Exp Psychol 2009, 62:2133-2140.

37. Bingham AF, Birch GG, de Graaf C, Behan JM, Perring KD: Sensory studies with sucrose-maltol mixtures. Chem Senses 1990, 15:447-456.

38. Green BG, Lim J, Osterhoff F, Blacher K, Nachtigal D: Taste mixture interactions: suppression, additivity, and the predominance of sweetness. Physiol Behav 2010, 101:731-737.

39. Kennedy O, Law C, Methven L, Mottram D, Gosney M: Investigating agerelated changes in taste and affects on sensory perceptions of oral nutritional supplements. Age Ageing 2010, 39:733-738.

40. Reed DR, Zhu G, Breslin PA, Duke FF, Henders AK, Campbell MJ, Montgomery GW, Medland SE, Martin NG, Wright MJ: The perception of quinine taste intensity is associated with common genetic variants in a bitter receptor cluster on chromosome 12. Hum Mol Genet 2010 19:4278-4285.

41. Murphy C, Cain WS: Taste and olfaction: independence vs interaction. Physiol Behav 1980, 24:601-605.

42. Saito S: Measurement method for olfaction. In Sensory and Perceptual Psychology Handbook. New edition. Edited by Oyama T, Imai S, Wake T. Tokyo: Seishin Shobo; 1994:1371-1382.

43. Toraya Confectionery: Types of wagashi. http://www.toraya-group.co.jp/ english/wagashi/types.html.

44. Green BG, Nachtigal D, Hammond S, Lim J: Enhancement of retronasal odors by taste. Chem Senses 2012, 37:77-86

45. Small DM, Voss J, Mak YE, Simmons KB, Parrish T, Gitelman D: Experiencedependent neural integration of taste and smell in the human brain. J Neurophysiol 2004, 92:1892-1903.

46. Stevenson RJ, Prescott J, Boakes RA: The acquisition of taste properties by odors. Learning and Motivation 1995, 26:433-455.

47. Stevenson RJ, Boakes RA, Prescott J: Changes in odor sweetness resulting from implicit learning of a simultaneous odor-sweetness association: an example of learned synesthesia. Learning and Motivation 1998, 29:113-132.

48. Levine AS, Kotz CM, Gosnell BA: Sugars: hedonic aspects, neuroregulation, and energy balance. Am J Clin Nutr 2003, 78:834S-842S.

49. Olszewski PK, Levine AS: Central opioids and consumption of sweet tastants: When reward outweighs homeostasis. Physiol Behav 2007, 91:506-512.

50. Ramirez I: Why do sugars taste good? Neurosci Biobehav Rev 1990, $14: 125-134$

doi:10.1186/2044-7248-2-26

Cite this article as: Gotow et al:: Retronasal aroma allows feature extraction from taste of a traditional Japanese confection. Flavour $20132: 26$ 